\title{
Aqueous-Alcohol-Processable High-Mobility Semiconducting Copolymers with Engineered Oligo(Ethylene Glycol) Side
} Chains

Boseok Kang, ${ }^{1,2 \dagger}$ Ziang Wu, ${ }^{3, \dagger}$ Min Je Kim, ${ }^{1}$ Han Young Woo ${ }^{3, *}$, Jeong Ho Cho ${ }^{4, *}$

${ }^{1}$ SKKU Advanced Institute of Nanotechnology, ${ }^{2}$ Department of Nano Engineering, Sungkyunkwan University, Suwon 16419, Republic of Korea.

${ }^{3}$ Department of Chemistry, Korea University, Seoul 02841, Republic of Korea

${ }^{4}$ Department of Chemical and Biomolecular Engineering, Yonsei University, Seoul 03722, Republic of Korea

*Address correspondence to jhcho94@yonsei.ac.kr (J.H.C) and hywoo@korea.ac.kr (H.Y.W)

${ }^{\dagger}$ B. Kang and Ziang Wu contributed equally to this work. 


\section{Details for Synthesis}

General synthesis. 4,7-Dibromo-5,6-difluoro-2,1,3-benzothiadiazole was purchased from Solarmer Energy Inc. Sodium hydride (NaH, 60\% dispersion in mineral oil), tetrakis(triphenylphosphine)palladium $(0)$ $\left(\mathrm{Pd}\left(\mathrm{PPh}_{3}\right)_{4}, 99 \%\right)$, tetraethyleneglycol monomethyl ether (2a), tris(dibenzylideneacetone)dipalladium $(0)$ $\left(\mathrm{Pd}_{2}(\mathrm{dba})_{3}, 97 \%\right)$ and tri(o-tolyl)phosphine $\left(\mathrm{P}(\mathrm{o}-\mathrm{tol})_{3}, 97 \%\right)$ were purchased from Sigma-Aldrich. 2,5bis(trimethylstannyl)thiophene and 5,5'-bis(trimethylstannyl)-2,2'-bithiophene ${ }^{[1]}, 2,5$ bis(trimethylstannyl)thieno[3,2-b]thiophene ${ }^{22}$, 5,5'-(2,5-difluoro-1,4-phenylene)bis(thiophene-5,2-diyl)) bis(trimethylstannane) $)^{[3]}, 5,5^{\prime}$-bis(trimethylstannyl)-3,3'-difluoro-2,2'-bithiophene ${ }^{[4]}$, compound $\mathbf{2 b}$, and $\mathbf{2 c}^{[5]}$ were synthesized according to previous literatures.

Monomer M1. 1a (3.0 mmol, $1.0 \mathrm{~g})$ and 2a (7.6 mmol, $1.6 \mathrm{~g})$ were dissolved in $50 \mathrm{~mL} N, N$ dimethylformamide (DMF). The mixture was cooled down to $0{ }^{\circ} \mathrm{C}$ and $\mathrm{NaH}(0.3 \mathrm{~g}, 7.6 \mathrm{mmol}, 60 \%$ dispersion in mineral oil) was added in portions. Then the mixture was allowed to warm to room temperature and stirred for 12 hours. Water $(5 \mathrm{~mL})$ was added to quench the reaction and all the solvent was removed by vacuum distillation. Water $(20 \mathrm{~mL})$ and dichloromethane (DCM) was added and the compound was extracted with DCM. The organic layer was combined and dried over anhydrous sodium sulfate $\left(\mathrm{Na}_{2} \mathrm{SO}_{4}\right)$. Solvent was removed under reduced pressure to obtain a brown liquid, which was purified by column chromatography on silica gel with a mixture of DCM:methanol (60:1-50:1 by volume) as eluent, yielding a yellow liquid (1.8 g, 84\%). ${ }^{1} \mathrm{H}$ NMR $\left(500 \mathrm{MHz}, \mathrm{CDCl}_{3}\right) \delta(\mathrm{ppm}) 4.41-4.34(\mathrm{~m}, 4 \mathrm{H}), 3.93-3.86(\mathrm{~m}$, $4 \mathrm{H}), 3.74-3.69(\mathrm{~m}, 4 \mathrm{H}), 3.69-3.60(\mathrm{~m}, 16 \mathrm{H}), 3.57-3.51(\mathrm{~m}, 4 \mathrm{H}), 3.37(\mathrm{~s}, 6 \mathrm{H}) .{ }^{13} \mathrm{C} \mathrm{NMR}\left(125 \mathrm{MHz}, \mathrm{CDCl}_{3}\right)$ $\delta(\mathrm{ppm}) 154.1,150.3,106.2,73.7,71.9,70.7,70.6,70.6,70.5,70.3,59.1$.

Monomer M2. The synthesis procedure was identical to that used for M1. 1a (6.1 mmol, $2 \mathrm{~g}), 2 \mathbf{b}(15.3$ mmol, $4.7 \mathrm{~g}) \mathrm{DMF}(80 \mathrm{~mL}), \mathrm{NaH}(0.61 \mathrm{~g}, 15.3 \mathrm{mmol}, 60 \%$ dispersion in mineral oil). Yield: $2.5 \mathrm{~g}, 46 \%$ (yellow liquid). ${ }^{1} \mathrm{H} \mathrm{NMR}\left(500 \mathrm{MHz}, \mathrm{CDCl}_{3}\right) \delta(\mathrm{ppm}) 4.23$ (d, 4H, J=6 Hz), 3.74-3.67 (m, 8H), 3.66-3.58 (m, $24 \mathrm{H}), 3.55-3.50(\mathrm{~m}, 8 \mathrm{H}), 3.36(\mathrm{~s}, 12 \mathrm{H}), 2.54-2.46(\mathrm{~m}, 2 \mathrm{H}) .{ }^{13} \mathrm{C} \mathrm{NMR}\left(125 \mathrm{MHz}, \mathrm{CDCl}_{3}\right) \delta(\mathrm{ppm}) 154.3$, $150.3,105.9,73.5,72.0,70.6,70.6,70.5,69.2,59.1,40.9$.

Monomer M3. The synthesis procedure was identical to that used for M1. 1a (3.0 mmol, $1 \mathrm{~g}), 2 \mathrm{c}(7.6 \mathrm{mmol}$, $3.0 \mathrm{~g})$, DMF (52 mL), NaH (0.30 g, $7.6 \mathrm{mmol}, 60 \%$ dispersion in mineral oil). Yield: $1.3 \mathrm{~g}, 40 \%$ (yellow liquid). ${ }^{1} \mathrm{H}$ NMR $\left(500 \mathrm{MHz}, \mathrm{CDCl}_{3}\right) \delta$ (ppm) 4.22(d, 4H, J=6.0 Hz), 3.78-3.60 (m, 48H), 3.56-3.50 (m, 8H), $3.36(\mathrm{~s}, 12 \mathrm{H}), 2.53-2.45(\mathrm{~m}, 2 \mathrm{H}) .{ }^{13} \mathrm{C} \mathrm{NMR}\left(125 \mathrm{MHz}, \mathrm{CDCl}_{3}\right) \delta(\mathrm{ppm}) 154.3,150.3,106.0,73.5,71.9$, $70.6,70.6,70.5,70.5,69.2,59.1,40.9$.

\section{Synthesis of Polymers}

PTBT. M1 (0.1 g, $0.142 \mathrm{mmol})$, 2,5-bis(trimethylstannyl)thiophene (0.058 g, $0.098 \mathrm{mmol}), \mathrm{Pd}\left(\mathrm{PPh}_{3}\right)_{4}(4$ $\mathrm{mol} \%)$, toluene $(1.1 \mathrm{~mL})$ and $\operatorname{DMF}(0.3 \mathrm{~mL})$ were combined in a microwave vial. The reaction mixture was heated at $80{ }^{\circ} \mathrm{C}$ for $3 \mathrm{~min}, 110^{\circ} \mathrm{C}$ for $3 \mathrm{~min}, 140{ }^{\circ} \mathrm{C}$ for $50 \mathrm{~min}$ and $150{ }^{\circ} \mathrm{C}$ for $55 \mathrm{~min}$ in a microwave reactor. Thereafter, 2 -tributylstannylthiophene ( 0.1 equiv) was added into the vial and the mixture was heated to $145{ }^{\circ} \mathrm{C}$ for $20 \mathrm{~min}$. 2-Bromothiophene ( 0.2 equiv) was then added and the mixture was again heated to $145^{\circ} \mathrm{C}$ for $20 \mathrm{~min}$. The reaction solution was precipitated into $300 \mathrm{~mL}$ hexane, filtered, and the crude polymer was purified by Soxhlet extraction with hexane, ether, and chloroform. The extracted portion in chloroform was precipitated into hexane, filtered, and dried under vacuum, yielding a deep-brown solid (0.08 g, 89\%). ${ }^{1} \mathrm{H}$ NMR (500 MHz, $\left.\mathrm{CDCl}_{3}\right) . \delta(\mathrm{ppm})$ 8.73-8.56 (br, 2H), 4.57-4.37 (br, 4H), 4.12-3.95 (br, $4 \mathrm{H}), 3.76-3.46(\mathrm{~m}, 24 \mathrm{H}), 3.34(\mathrm{~s}, 6 \mathrm{H})$.

PTTBT. M2 (0.219 g, $0.240 \mathrm{mmol})$, 2,5-bis(trimethylstannyl)thieno[3,2-b]thiophene (0.112 g, 0.240 
$\mathrm{mmol}), \mathrm{Pd}_{2}(\mathrm{dba})_{3}(2 \mathrm{~mol} \%), \mathrm{P}(\mathrm{o}-\mathrm{tol})_{3}(16 \mathrm{~mol} \%)$ and toluene $(2.4 \mathrm{~mL})$ were combined in a microwave vial. The reaction mixture was heated at $80^{\circ} \mathrm{C}$ for $3 \mathrm{~min}, 110^{\circ} \mathrm{C}$ for $3 \mathrm{~min}, 140{ }^{\circ} \mathrm{C}$ for $50 \mathrm{~min}$ and $150{ }^{\circ} \mathrm{C}$ for 55 min in a microwave reactor. Thereafter, 2 -tributylstannylthiophene ( 0.1 equiv) was added into the vial and the mixture was heated to $145^{\circ} \mathrm{C}$ for $20 \mathrm{~min}$. 2-Bromothiophene ( 0.2 equiv) was then added and the mixture was again heated to $145^{\circ} \mathrm{C}$ for $20 \mathrm{~min}$. The reaction solution was precipitated by pouring into $300 \mathrm{~mL}$ hexane, filtered, and further purified by Soxhlet extraction with hexane, ether, and chloroform. The extracted portion in chloroform was precipitated into hexane, filtered, and dried under vacuum, yielding a deep-blue solid (0.18 g, 85\%). ${ }^{1} \mathrm{H}$ NMR (500 MHz, $\left.\mathrm{CDCl}_{3}\right) . \delta(\mathrm{ppm})$ 8.79-8.64 (br, 2H), 4.46-4.12 (br, 4H), 3.73-3.46 (br, 40H), 3.33 (s, 12H), 2.75-2.66 (br, 2H).

P2TBT. The synthesis procedure was identical to that used for PTBT. M2 $(0.1 \mathrm{~g}, 0.116 \mathrm{mmol}), 5,5^{\prime}-$ bis(trimethylstannyl)-2,2'-bithiophene (0.055 g, $0.116 \mathrm{mmol}), \mathrm{Pd}\left(\mathrm{PPh}_{3}\right)_{4}(4 \mathrm{~mol} \%)$, toluene $(1.0 \mathrm{~mL})$ and DMF (0.22 mL). Deep brown solid (Yield: $0.083 \mathrm{~g}, 80 \%)$. ${ }^{1} \mathrm{H}$ NMR (500 MHz, $\left.\mathrm{CDCl}_{3}\right) . \delta(\mathrm{ppm})$ 8.33-8.22 (br, 2H), 7.47-7.37 (br, 2H), 4.32-4.19 (br, 4H), 3.71-3.44 (m, 40H), 3.33 (s, 12H), 2.65-2.57 (br, 2H).

P2F2TBT. The synthesis procedure was identical to that used for PTTBT. M2 $(0.255 \mathrm{~g}, 0.280 \mathrm{mmol}), 5^{\prime}$ bis(trimethylstannyl)-3,3'-difluoro-2,2'-bithiophene (0.148 g, $0.280 \mathrm{mmol}), \mathrm{Pd}_{2}(\mathrm{dba})_{3}(2 \mathrm{~mol} \%), \mathrm{P}(\mathrm{o}-\text { tol })_{3}$ $(16 \mathrm{~mol} \%)$ and toluene $(2.0 \mathrm{~mL})$. Deep blue solid (Yield: $0.22 \mathrm{~g}, 83 \%) .{ }^{1} \mathrm{H} \mathrm{NMR}\left(500 \mathrm{MHz}, \mathrm{CDCl}_{3}\right) . \delta$ (ppm) 8.64-8.20 (br, 2H), 4.58-4.10 (br, 4H), 4.02-3.45 (m, 40H), 3.33 (s, 12H), 3.01-2.70 (br, 2H).

PBT2T2FB. The synthesis procedure was identical to that used for PTTBT. M3 $(0.251 \mathrm{~g}, 0.231 \mathrm{mmol})$, 5,5'-(2,5-difluoro-1,4-phenylene)bis(thiophene-5,2-diyl))bis(trimethylstannane)) (0.140 g, $0.231 \mathrm{mmol})$, $\mathrm{Pd}_{2}(\mathrm{dba})_{3}(2 \mathrm{~mol} \%), \mathrm{P}(\mathrm{o}-\mathrm{tol})_{3}(16 \mathrm{~mol} \%)$ and toluene $(2.0 \mathrm{~mL})$. Deep brown solid (Yield: $\left.0.24 \mathrm{~g}, 87 \%\right)$. ${ }^{1} \mathrm{H}$ NMR (500 MHz, $\mathrm{CDCl}_{3}$ ). $\delta$ (ppm) 8.34-8.26 (br, 2H), 7.72-7.65 (br, 2H), 7.63-7.54 (br, 2H), 4.33-4.20 (br, $4 \mathrm{H}), 3.70-3.53(\mathrm{~m}, 48 \mathrm{H}), 3.52-3.47(\mathrm{~m}, 8 \mathrm{H}), 3.34(\mathrm{~s}, 12 \mathrm{H}), 2.63-2.54$ (br, 2H). 


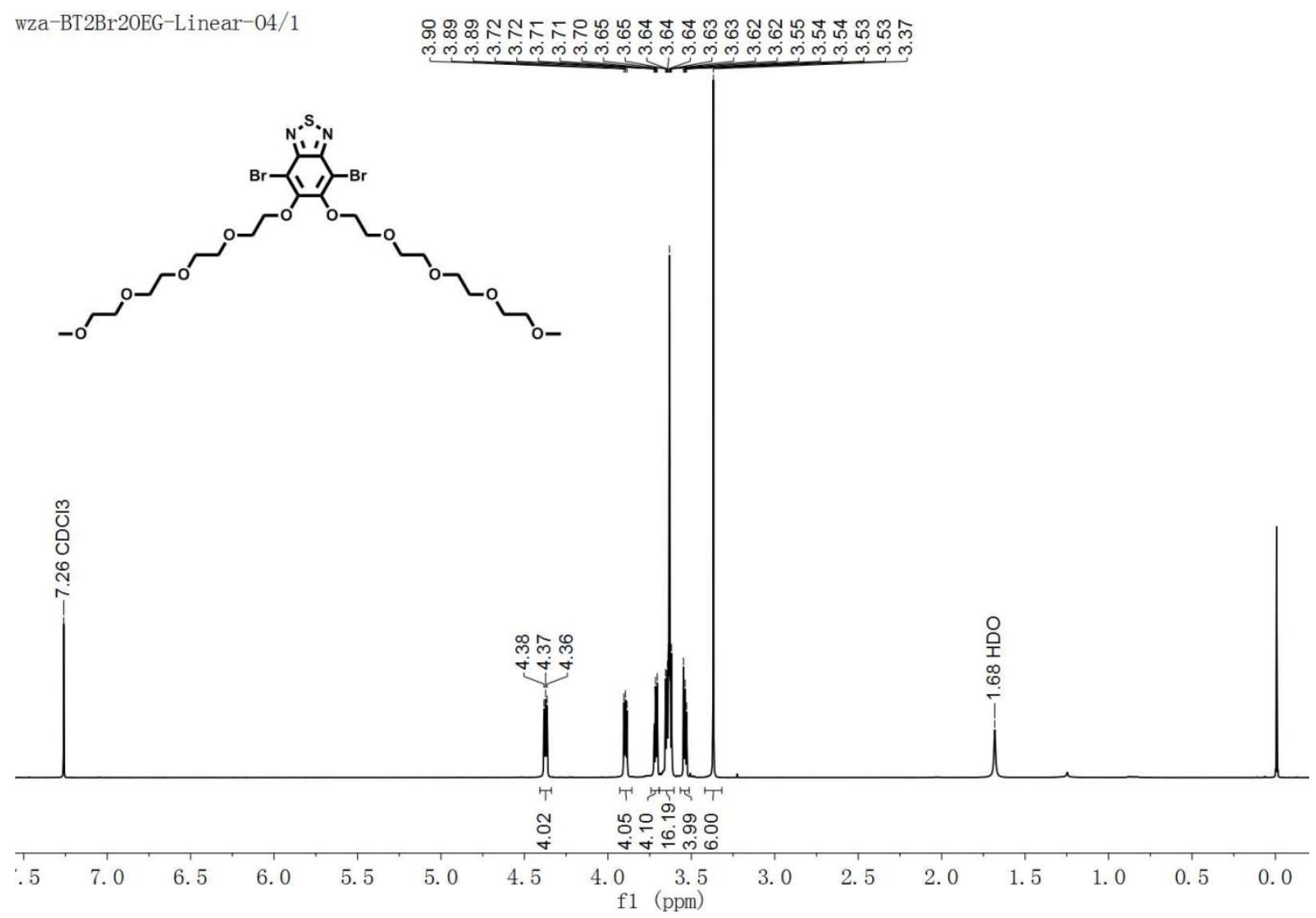

Figure S1. ${ }^{1} \mathrm{H}$ NMR spectrum of M1.

wza-BT2Br20EG-Linear-04-C/2

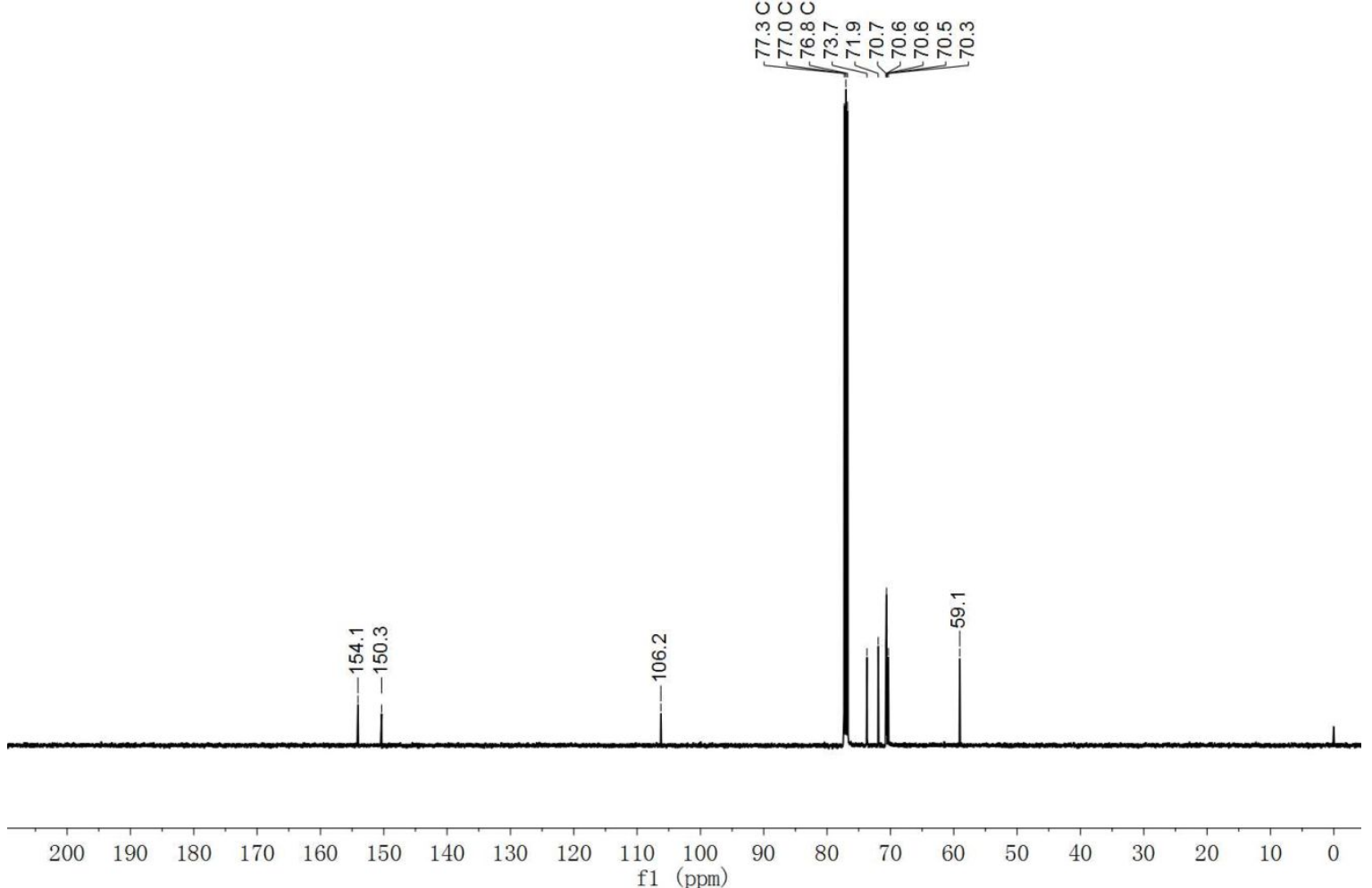

Figure S2. ${ }^{13} \mathrm{C}$ NMR spectrum of M1. 


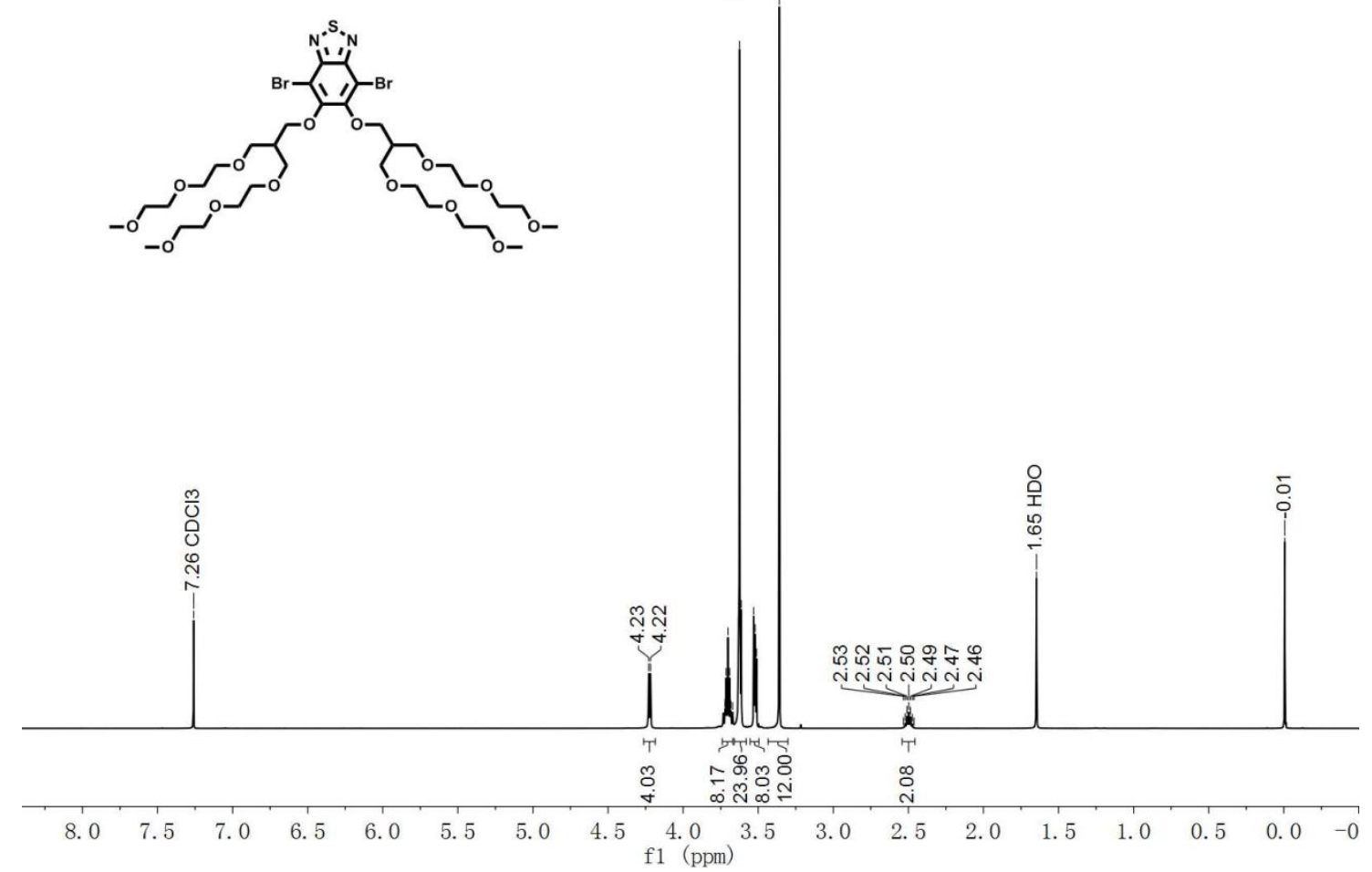

Figure S3. ${ }^{1} \mathrm{H}$ NMR spectrum of $\mathbf{M} 2$.

wza-BT2Br20EG-branch-03-C/1

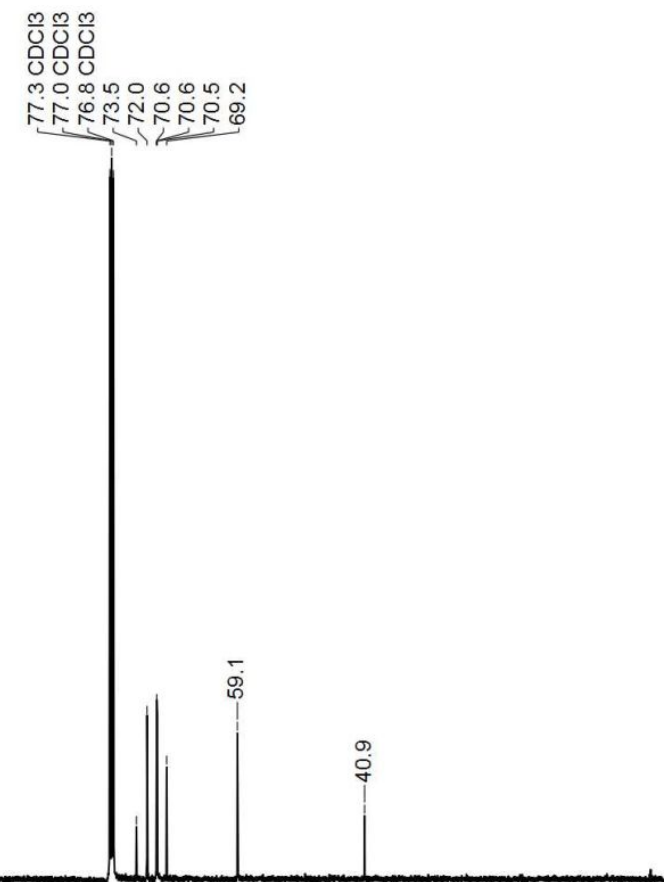

\begin{tabular}{rlllllllllllllllllllllllllll}
\hline 10 & 200 & 190 & 180 & 170 & 160 & 150 & 140 & 130 & 120 & 110 & 100 & 90 & 80 & 70 & 60 & 50 & 40 & 30 & 20 & 10 & 0
\end{tabular}

Figure S4. ${ }^{13} \mathrm{C}$ NMR spectrum of $\mathbf{M} 2$. 

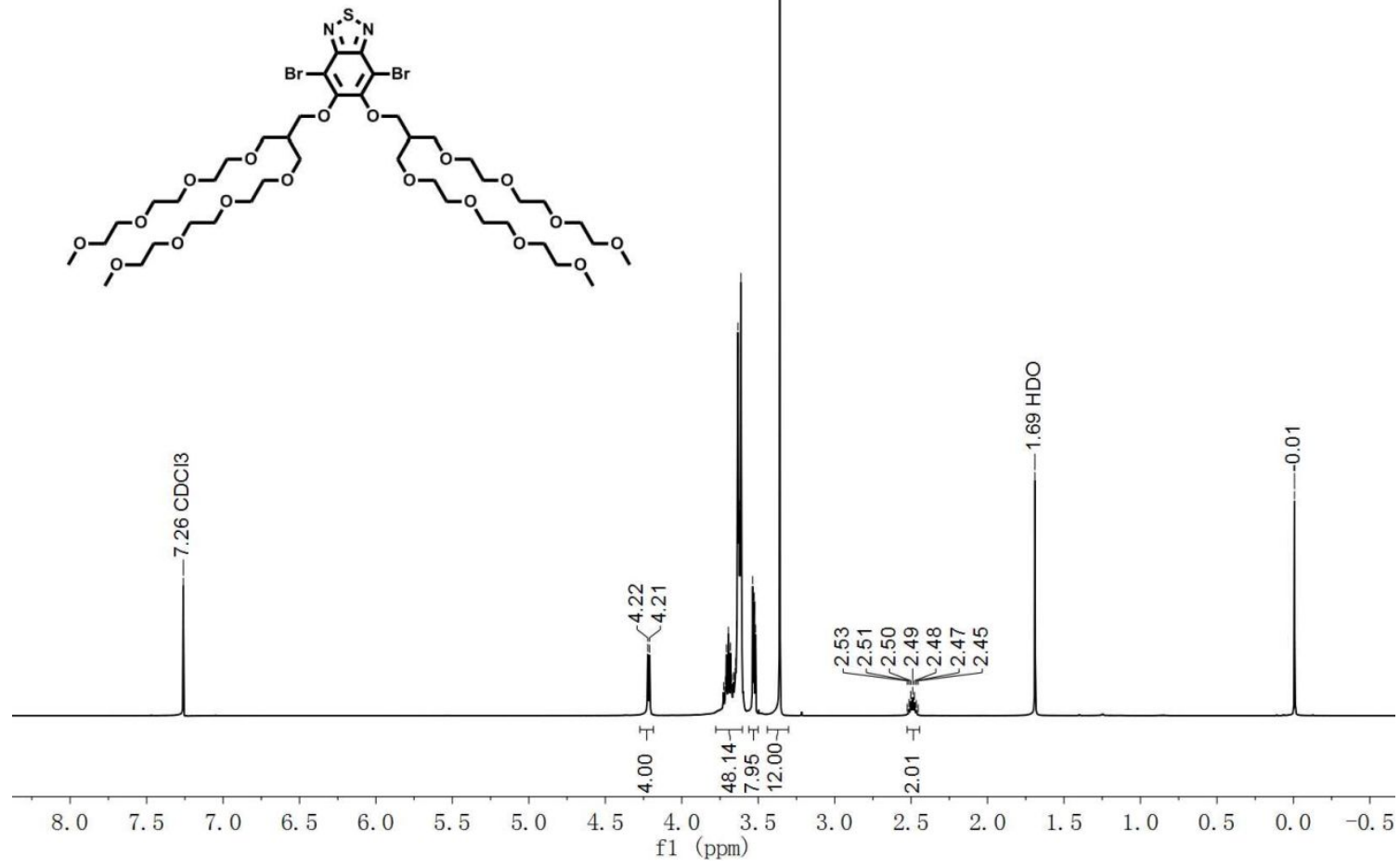

Figure S5. ${ }^{1} \mathrm{H}$ NMR spectrum of M3.

wza-BT2Br20EG-branch-04-C/2

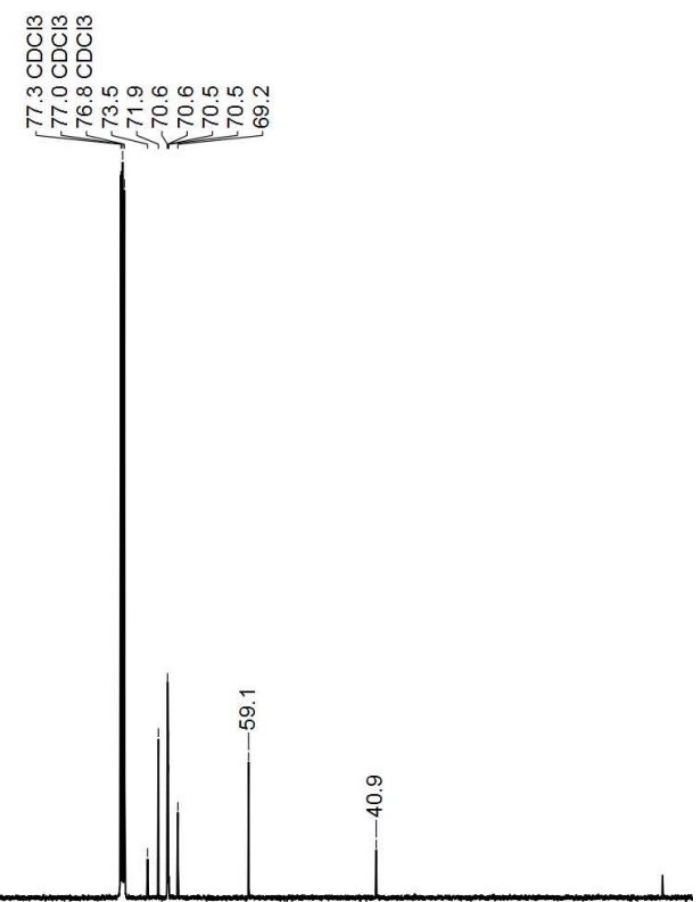

$\begin{array}{lllllllllllllllllllll}200 & 190 & 180 & 170 & 160 & 150 & 140 & 130 & 120 & \begin{array}{c}110 \\ \mathrm{f} 1\end{array}\left(\begin{array}{c}100 \\ (\mathrm{ppm})\end{array}\right. & 90 & 80 & 70 & 60 & 50 & 40 & 30 & 20 & 10 & 0\end{array}$

Figure S6. ${ }^{13} \mathrm{C}$ NMR spectrum of M3. 

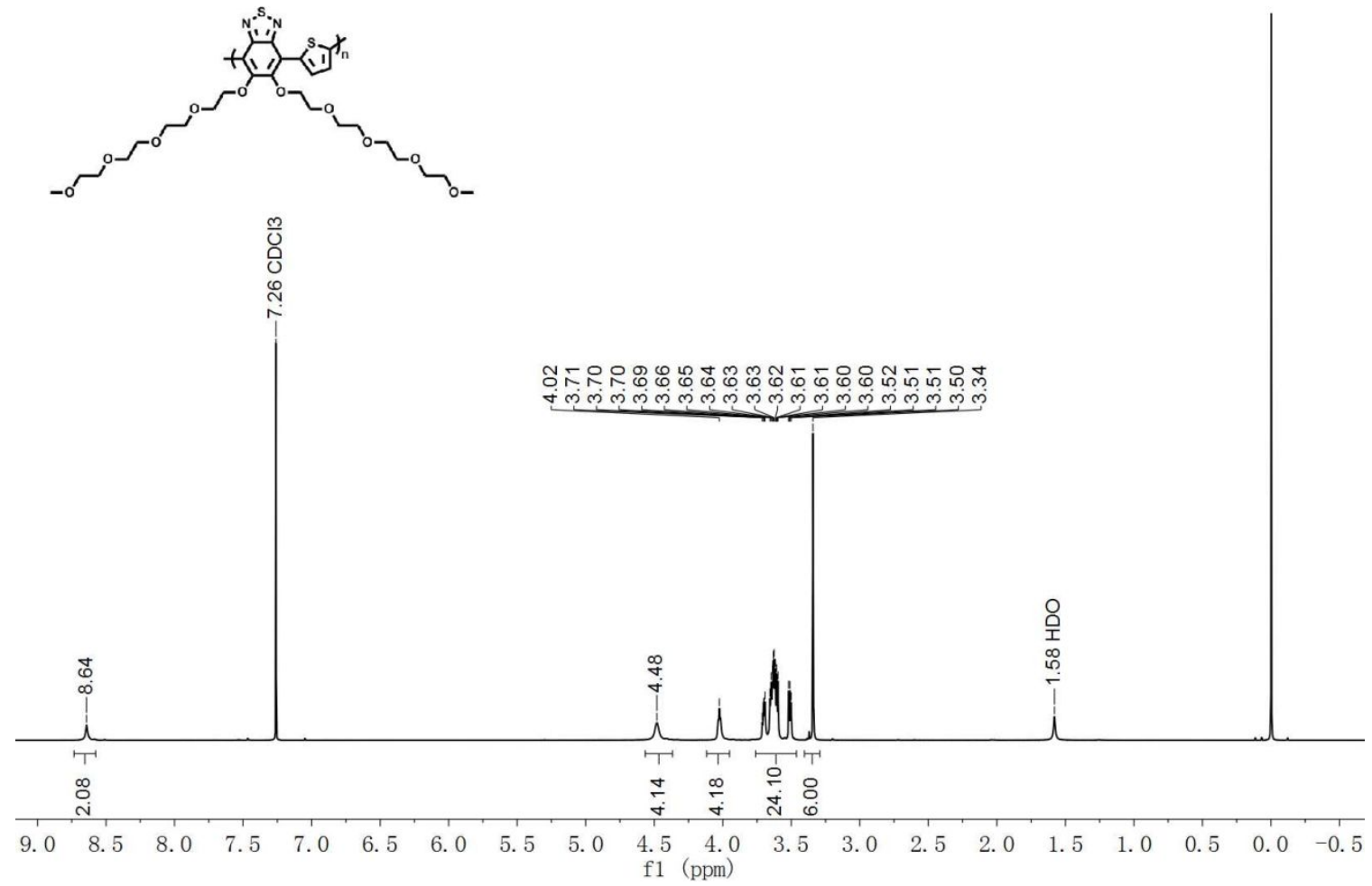

Figure S7. ${ }^{1} \mathrm{H}$ NMR spectrum of PTBT.

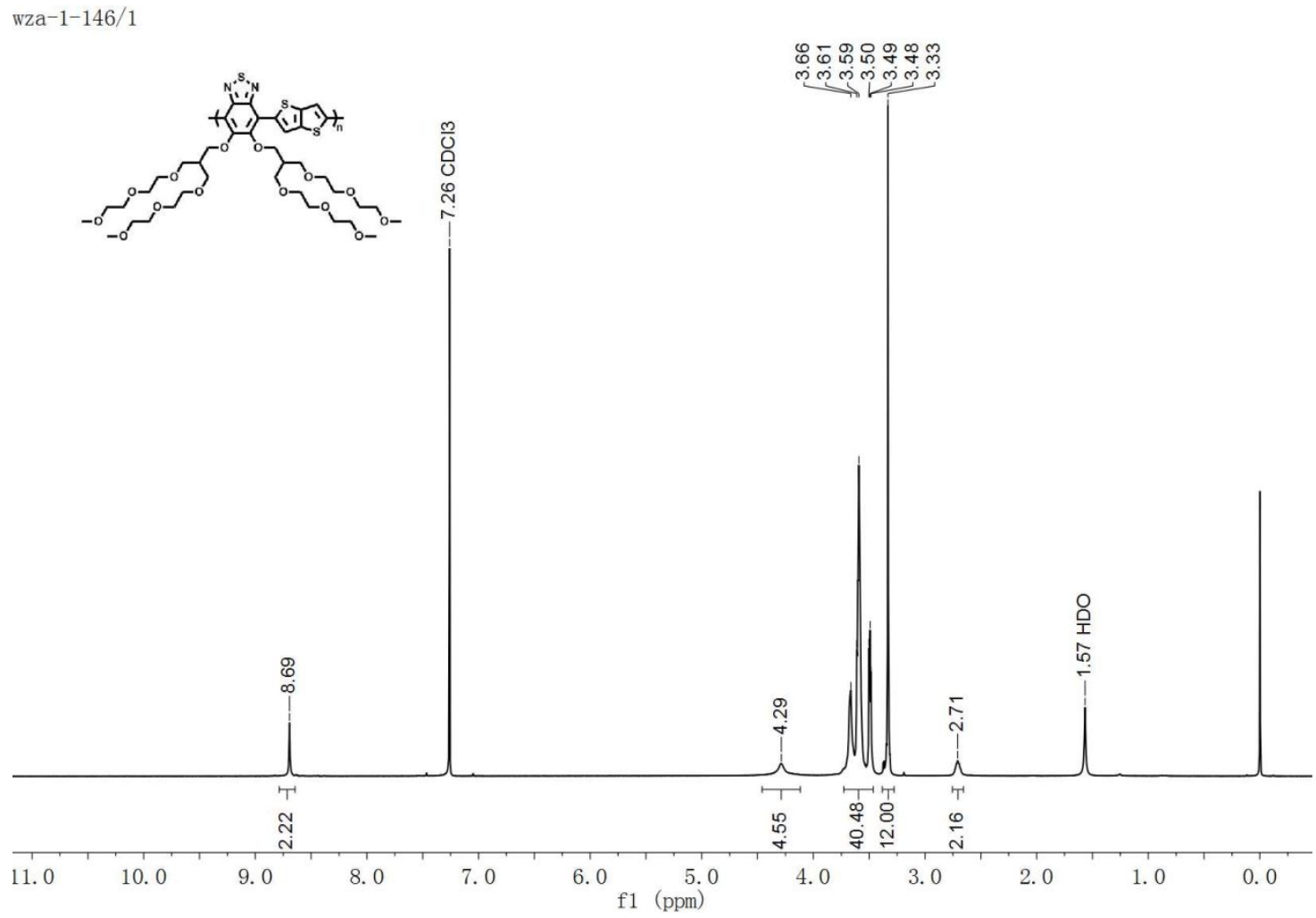

Figure S8. ${ }^{1} \mathrm{H}$ NMR spectrum of PTTBT. 


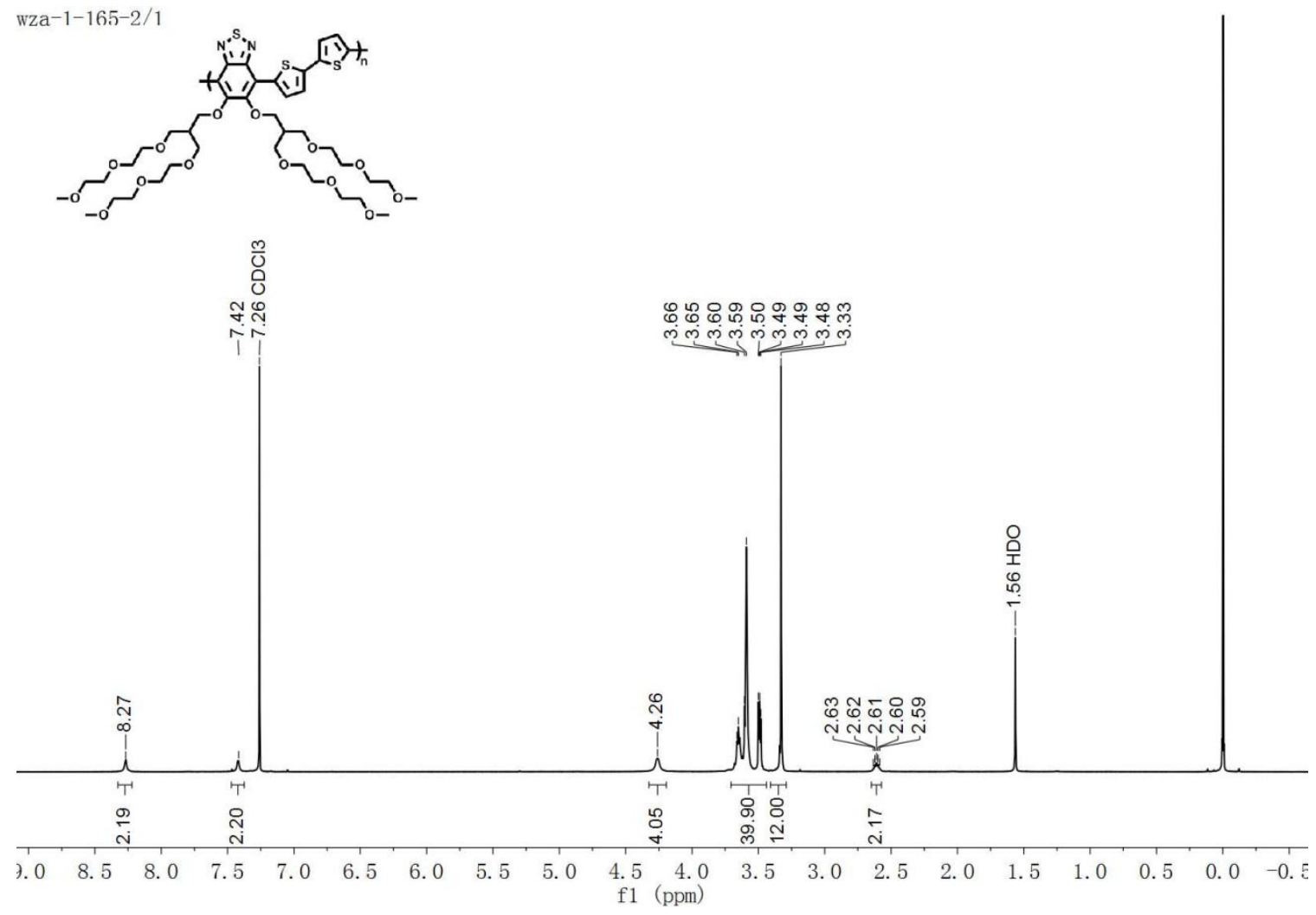

Figure S9. ${ }^{1} \mathrm{H}$ NMR spectrum of P2TBT.

wza-1-144/1

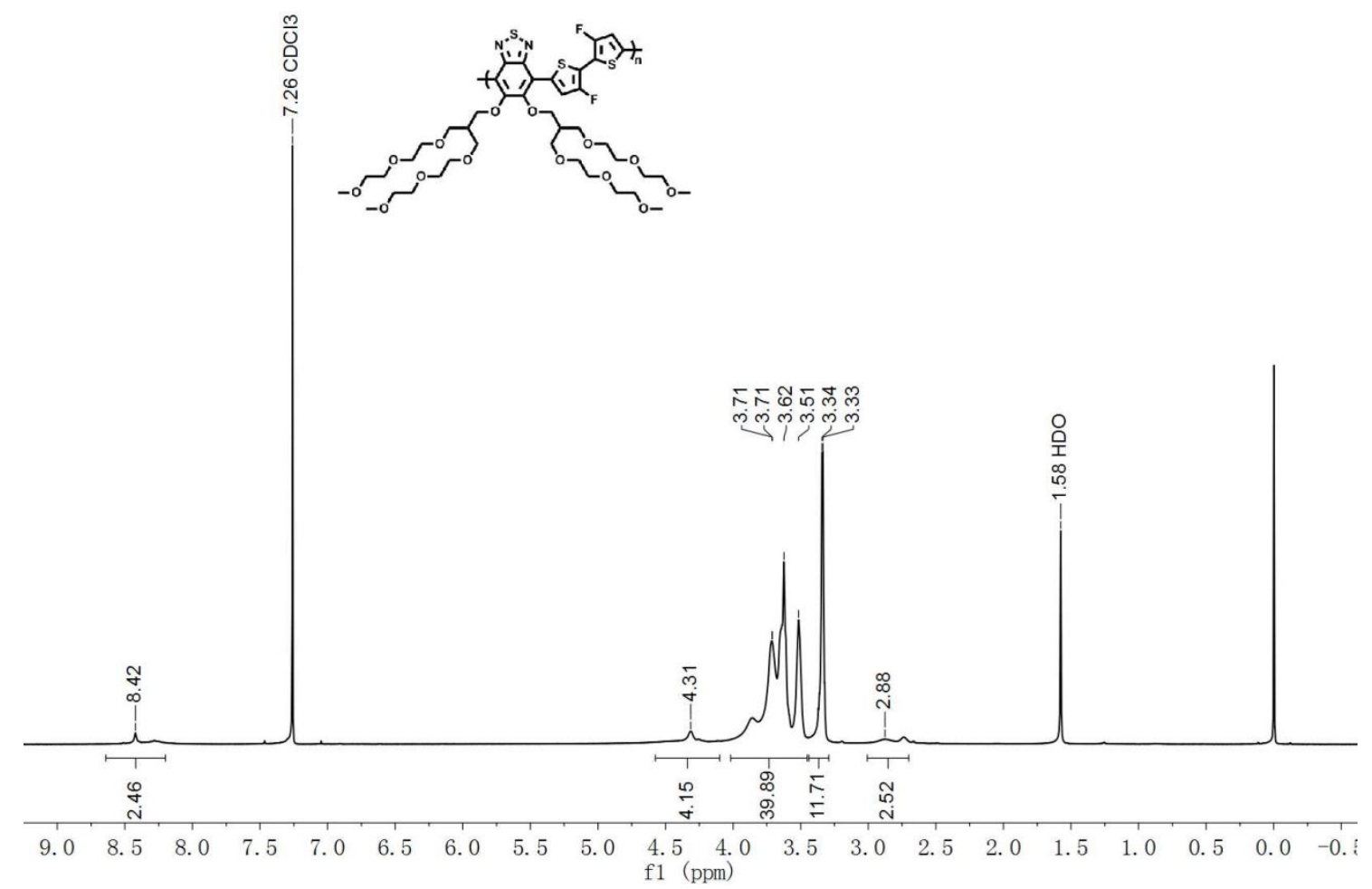

Figure S10. ${ }^{1} \mathrm{H}$ NMR spectrum of P2F2TBT. 


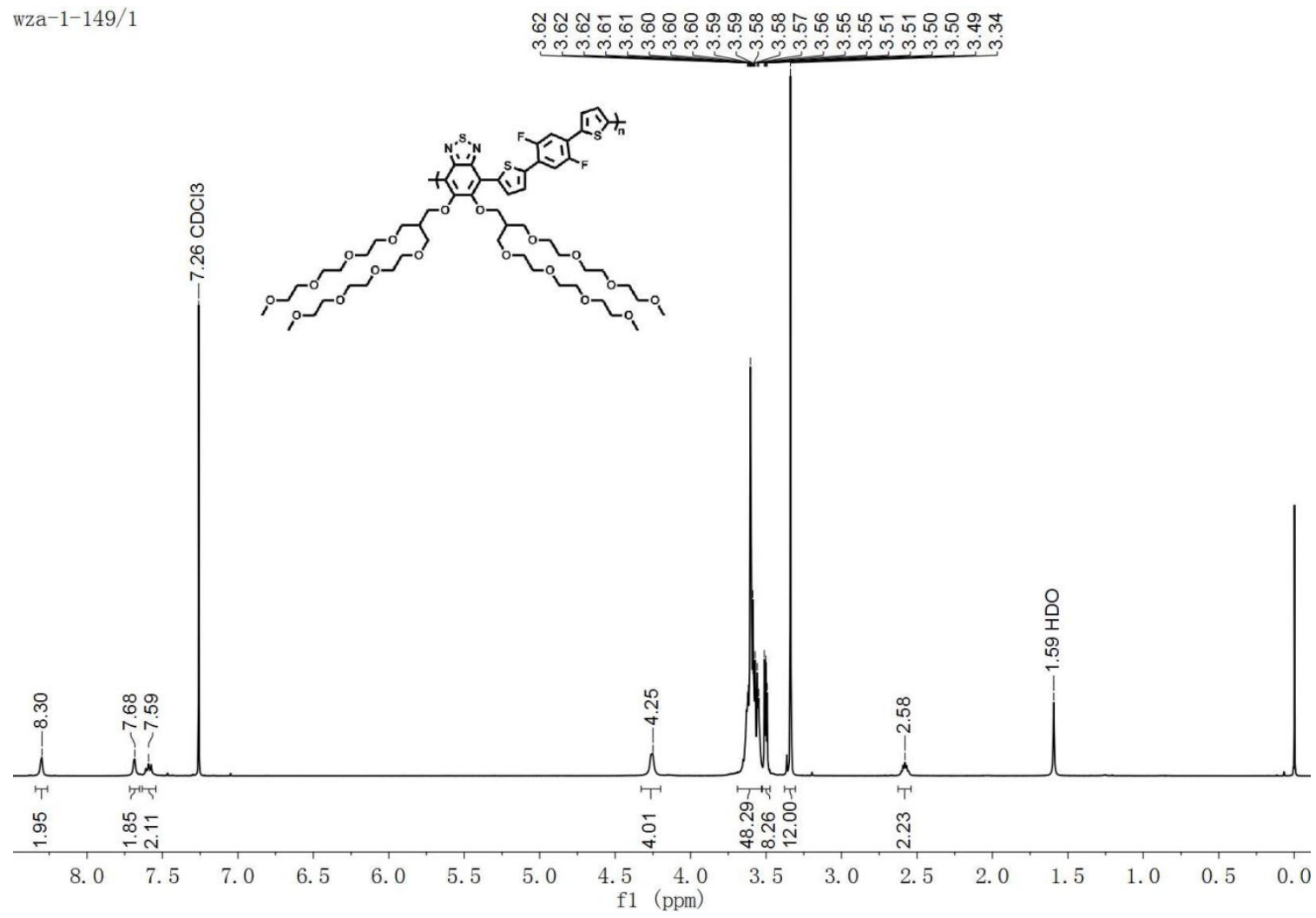

Figure S11. ${ }^{1} \mathrm{H}$ NMR spectrum of PBT2T2FB. 


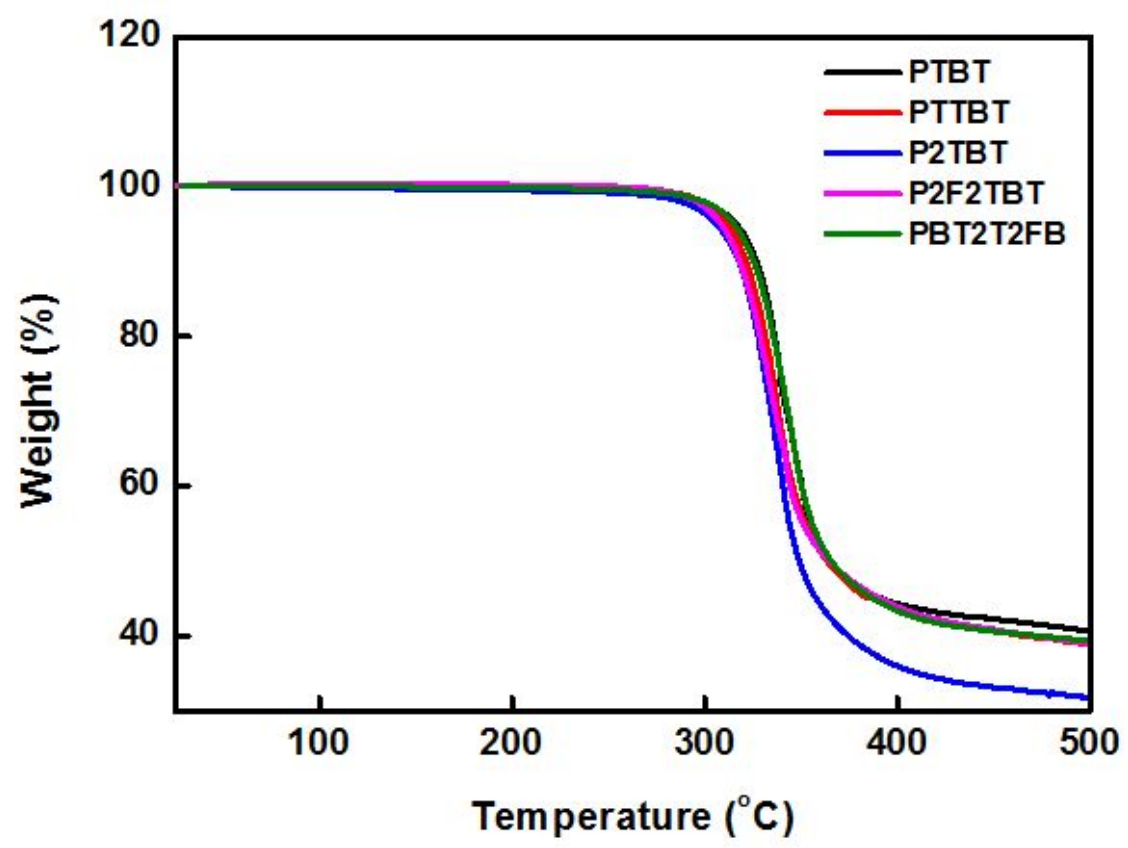

Figure S12. TGA thermograms of the copolymers. 

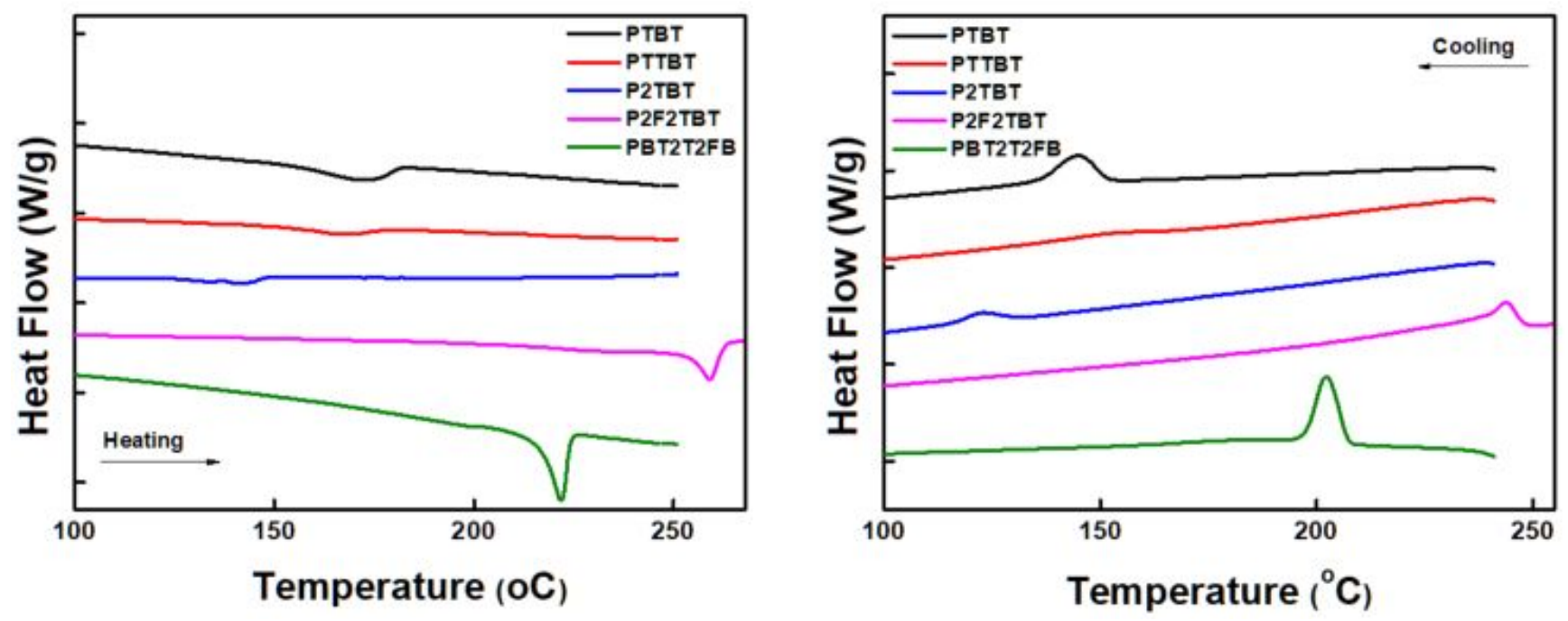

Figure S13. DSC thermograms of the copolymers. 


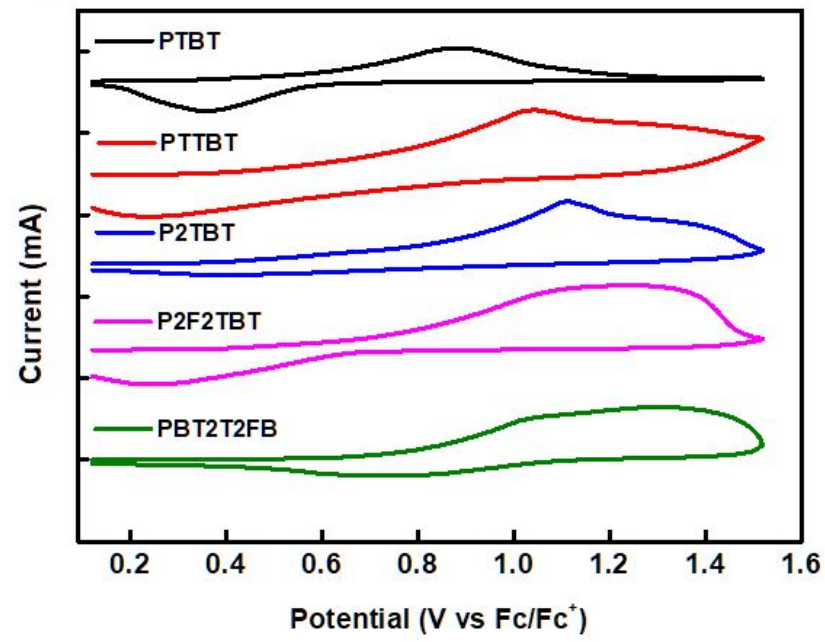

b

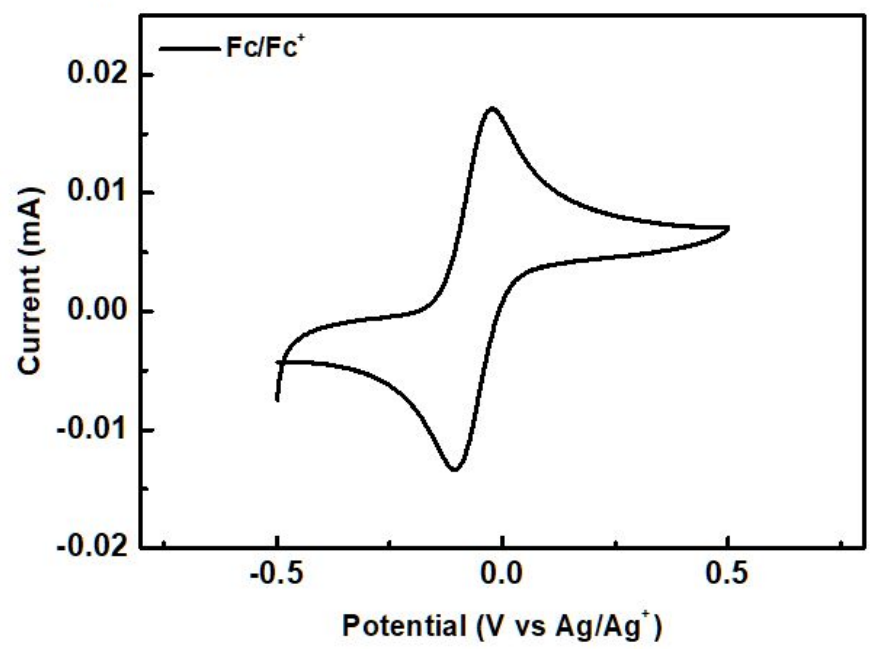

Figure 14. Cyclic voltammograms of five copolymer films relative to the $\mathrm{Fc} / \mathrm{Fc}^{+}$reference (a) and ferrocene (b). 


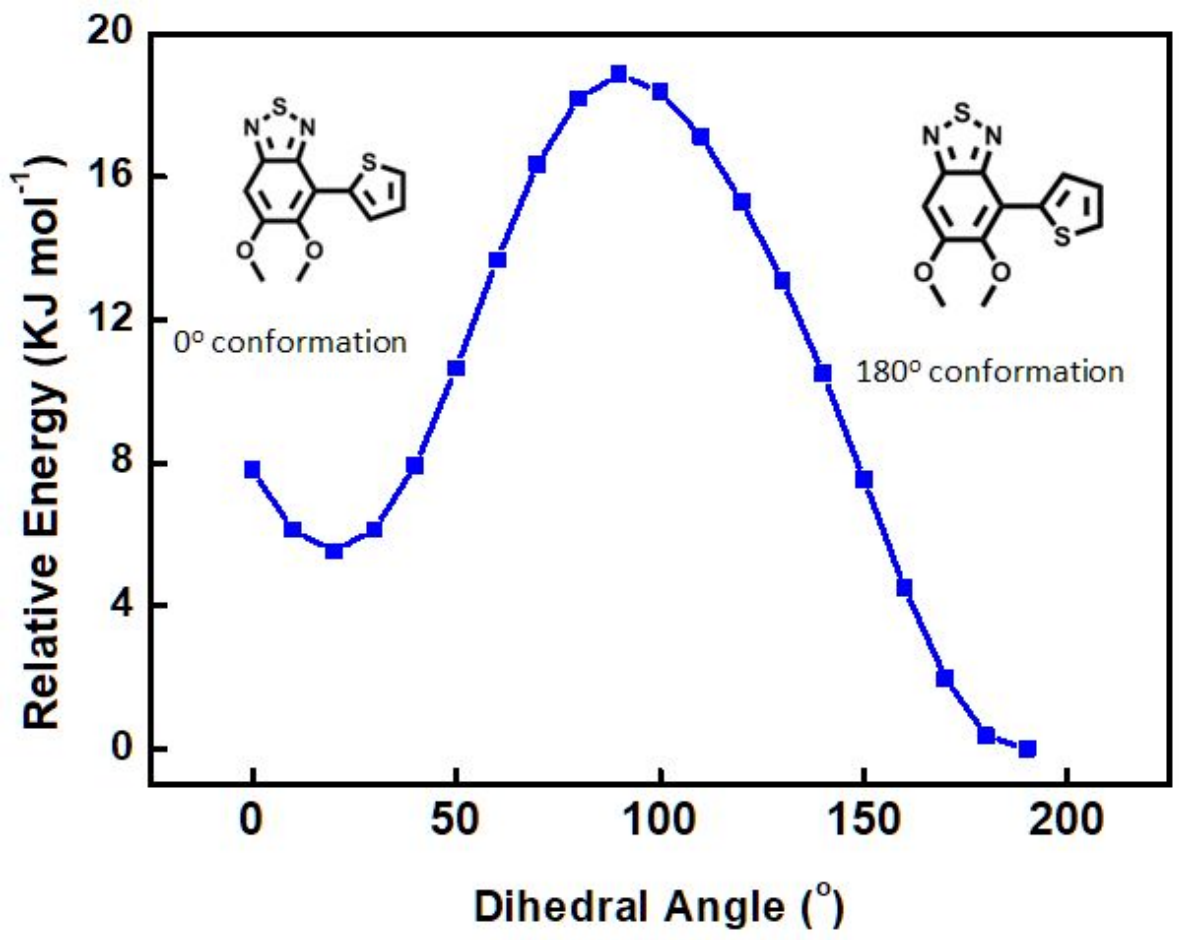

Figure S15. DFT-calculated torsional profiles of thiophene-BT linkage 


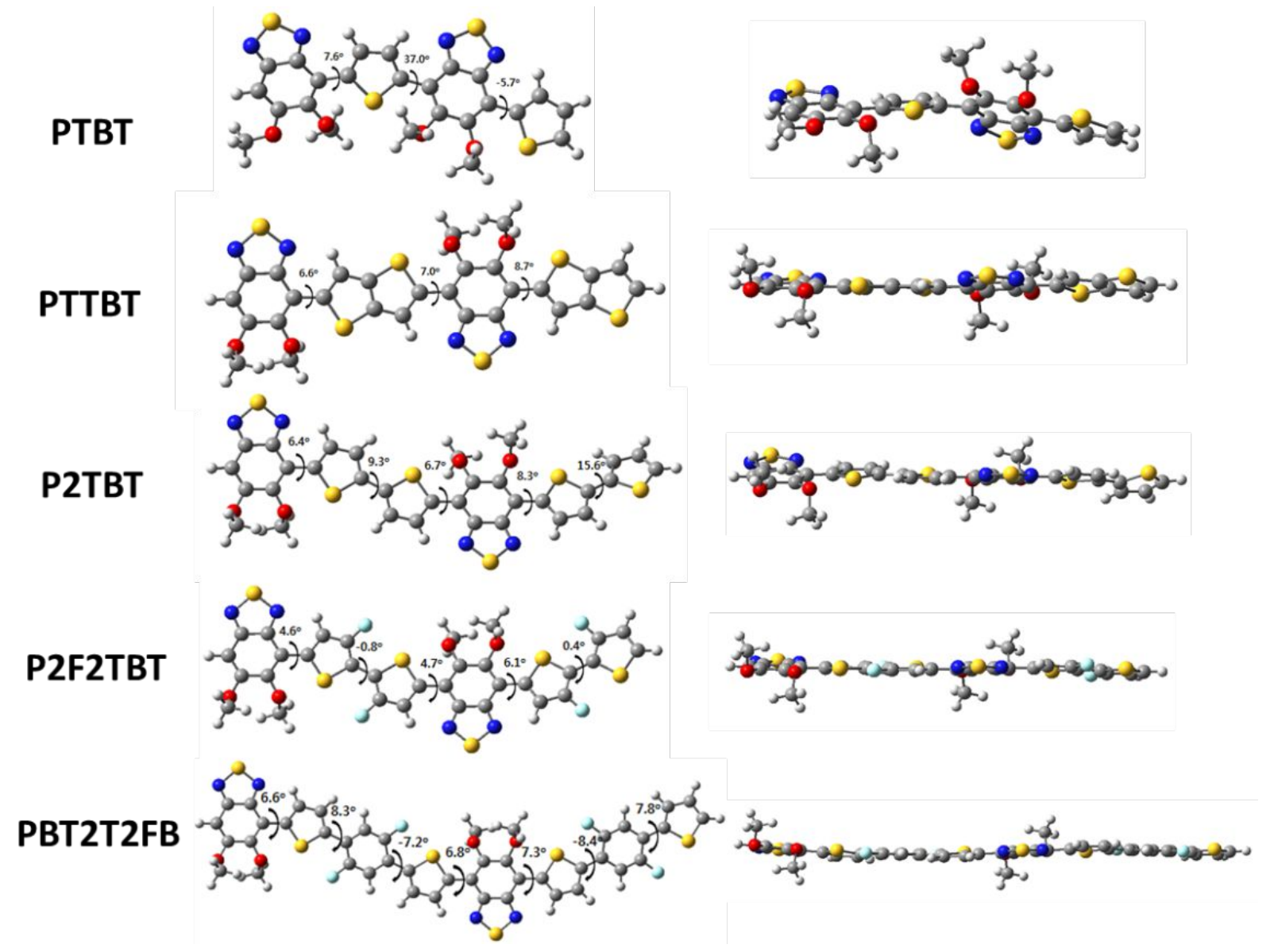

Figure S16. Energy-minimized geometries of five copolymers based on two repeat units. 


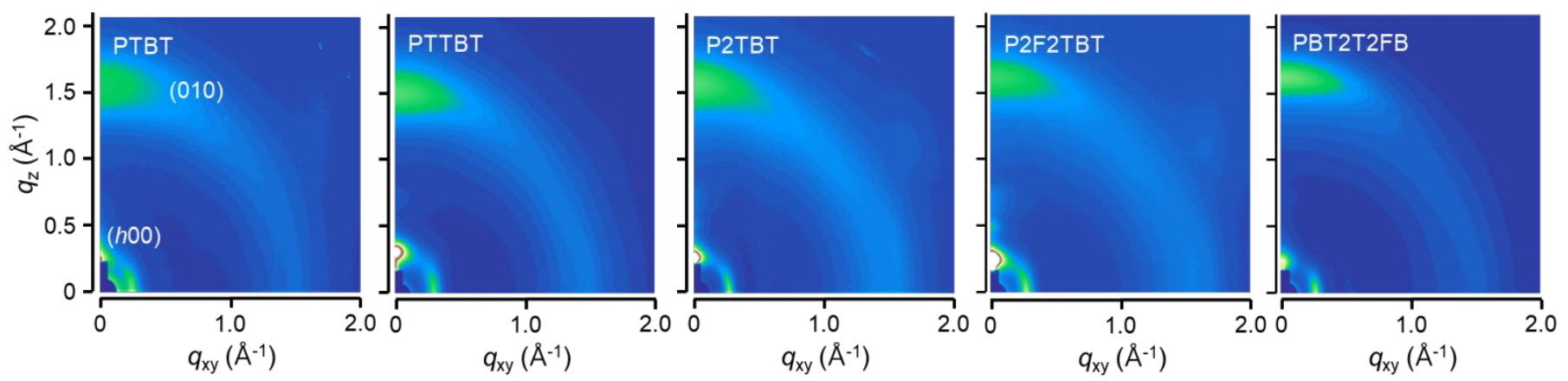

Figure S17. 2D GIXD patterns of as-spun polymer thin films. 

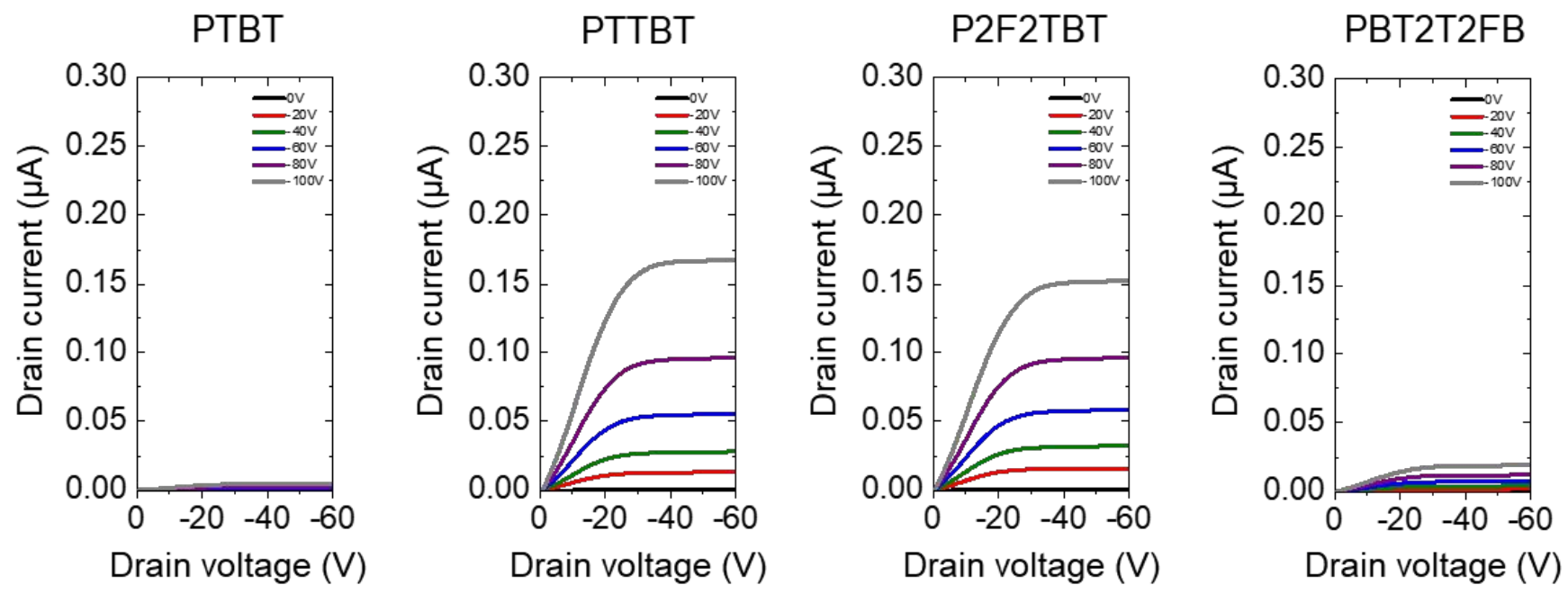

Figure S18. Output characteristics of bottom-gate top-contact PFETs. 
a
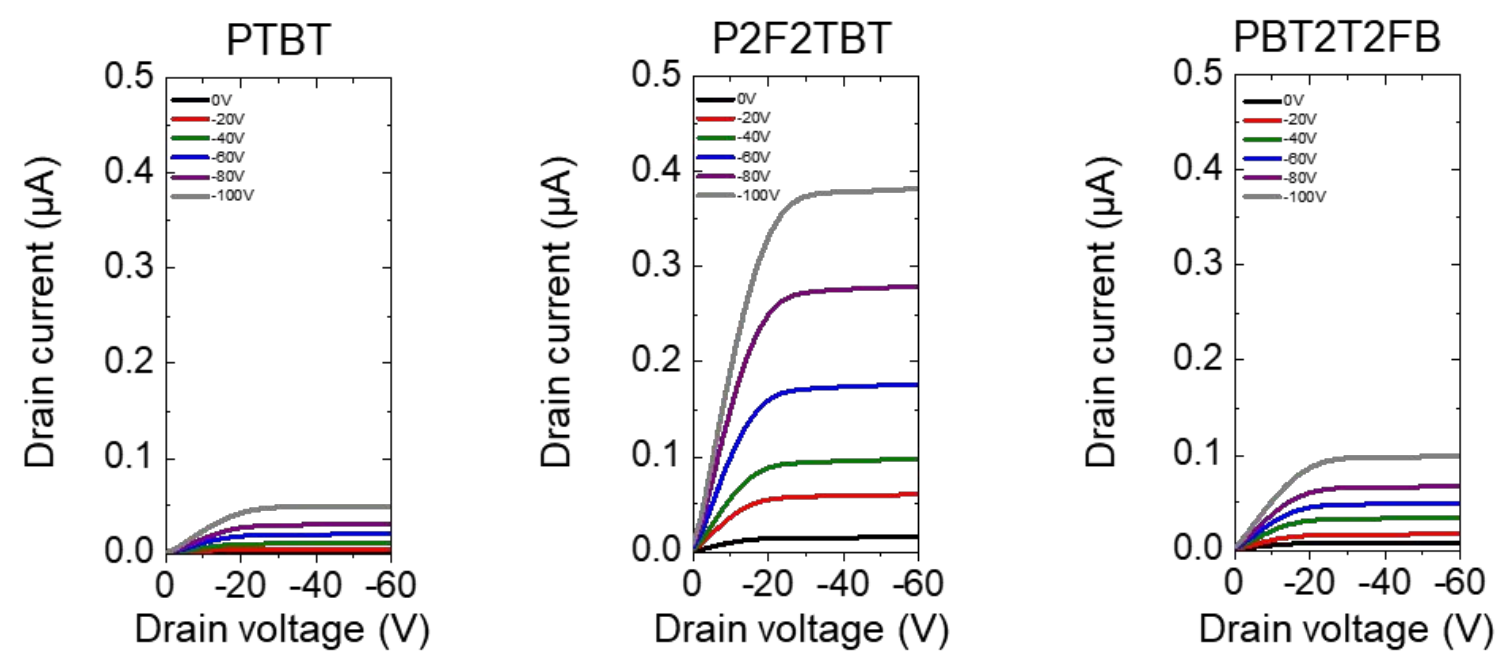

b
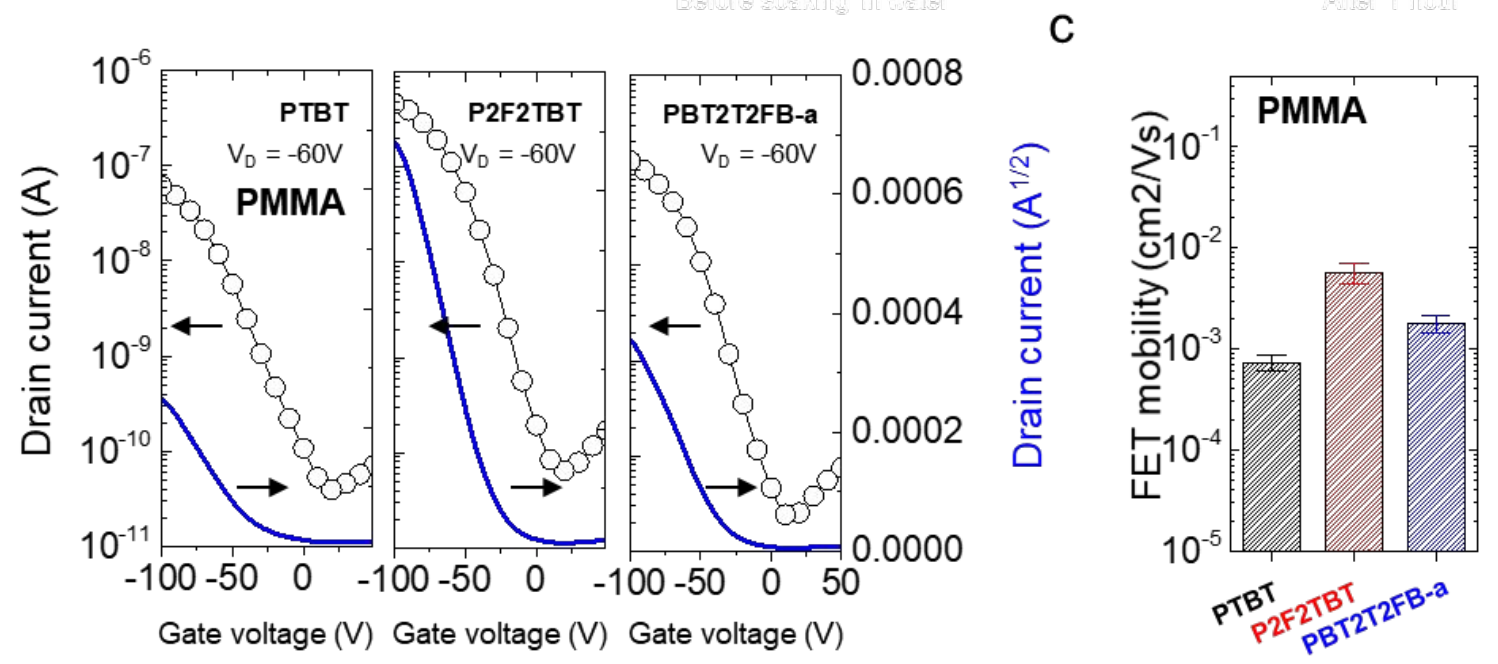

Figure S19. (a) Output characteristics, (b) transfer characteristics, and (c) summary of mobilities for topgate bottom-contact PFETs prepared with PMMA gate dielectric. 


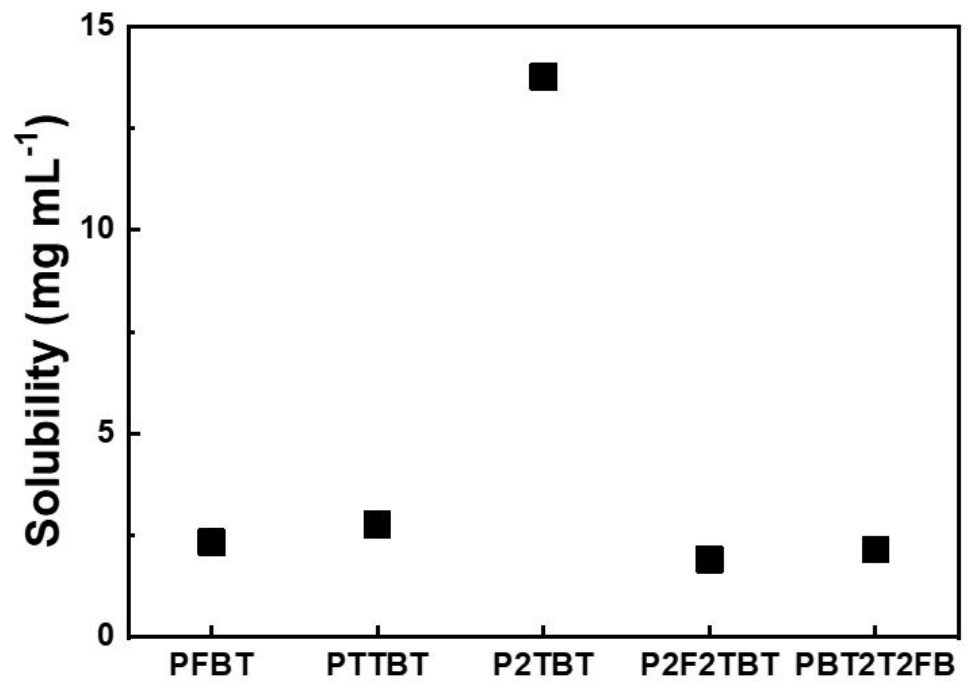

Figure S20. Room-temperature solubility of copolymers in an ethanol/water mixed solvent (9/1 vol) 

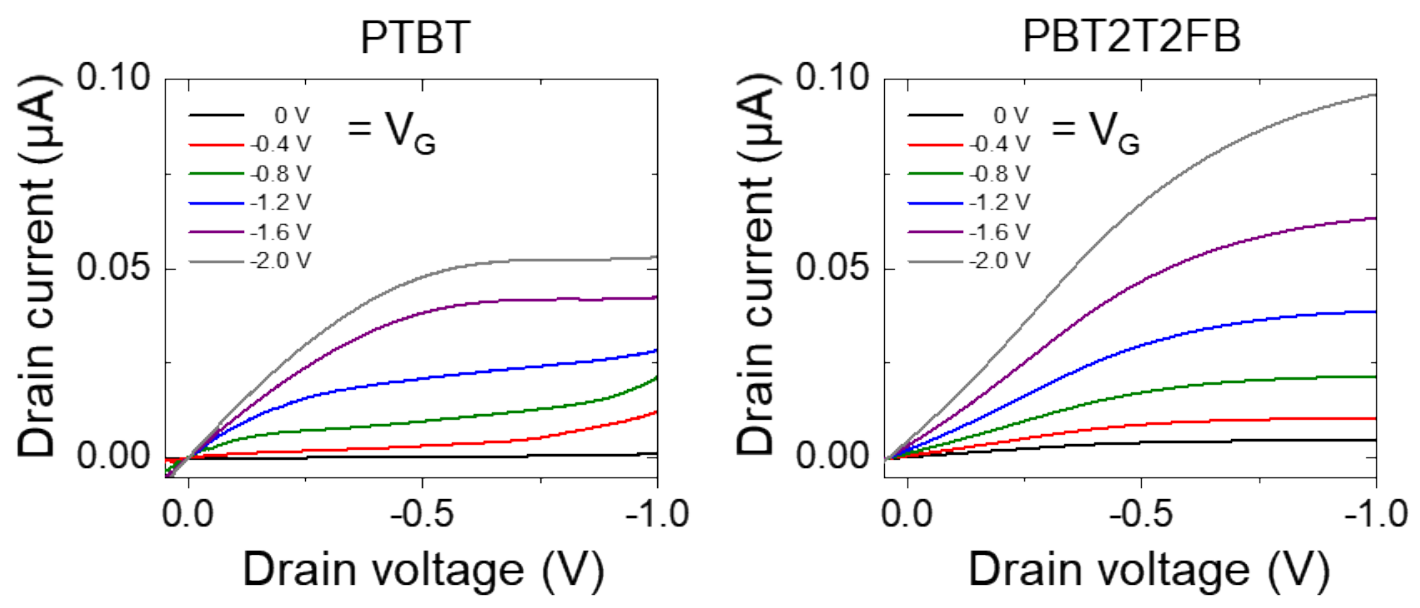

Figure S21. Output characteristics of top-gate bottom-contact PFETs prepared with PSSH gate dielectric. 
Table S1. 2D GIXD crystallographic information of the copolymer thin films annealed at $200{ }^{\circ} \mathrm{C}$.

\begin{tabular}{|c|c|c|c|c|c|c|}
\hline \hline \multicolumn{1}{|c|}{ Crystallographic parameters } & PTBT & PTTBT & P2TBT & P2F2TBT & PBT2T2FB \\
\hline \multirow{4}{*}{$\begin{array}{c}\text { Lamelar } \\
\text { stacking }\end{array}$} & $q\left(\AA^{-1}\right)$ & 0.27074 & 0.31144 & 0.32393 & 0.28285 & 0.24882 \\
\cline { 2 - 7 } & $d$-spacing $(\AA)$ & 23.2 & 20.2 & 19.4 & 22.2 & 25.3 \\
\cline { 2 - 7 } & FWHM $\left(\AA^{-1}\right)$ & 0.02624 & 0.03167 & 0.03168 & 0.03081 & 0.04971 \\
\hline \multirow{4}{*}{$\pi-\pi$} \\
\cline { 2 - 7 } stacking & Correlation length $(\AA)^{\mathrm{a}}$ & 431.1 & 357.2 & 357.1 & 367.2 & 227.6 \\
\cline { 2 - 7 } & $d$-spacing $(\AA)$ & 1.76245 & 1.57576 & 1.62248 & 1.66234 & 1.69351 \\
\cline { 2 - 7 } & FWHM $\left(\AA^{-1}\right)$ & 0.05402 & 0.37224 & 0.1167 & 0.27262 & 0.11678 \\
\cline { 2 - 7 } & Correlation length $(\AA)$ & 212.0 & 30.7 & 97.9 & 41.9 & 98.0 \\
\hline
\end{tabular}

${ }^{a}$ The correlation length is determined from the fullwidth-at-half-maximum (FWHM) of X-ray diffraction using the Scherrer equation. 
Table S2. Performance parameters of BGTC PFETs fabricated on $\mathrm{SiO}_{2}$ dielectrics.

\begin{tabular}{|c|c|c|c|c|}
\hline & & \multicolumn{3}{|c|}{ Annealing temperature } \\
\hline & & $25^{\circ} \mathrm{C}$ & $100^{\circ} \mathrm{C}$ & $200^{\circ} \mathrm{C}$ \\
\hline \multirow{3}{*}{ PTBT } & $V_{\mathrm{TH}}$ & $-7.4( \pm 1.2) \mathrm{V}$ & $-8.5( \pm 1.6) \mathrm{V}$ & $-14.7( \pm 4.5) \mathrm{V}$ \\
\hline & $I_{\mathrm{ON}} / I_{\mathrm{OFF}}$ & $39.0( \pm 4.4)$ & $83.9( \pm 8.7)$ & $121.9( \pm 13.8)$ \\
\hline & $\mu_{\mathrm{h}}$ & $8.6( \pm 2.0) \times 10^{-6}$ & $1.9( \pm 4.4) \times 10^{-5}$ & $4.2( \pm 1.0) \times 10^{-5}$ \\
\hline \multirow{3}{*}{ PTTBT } & $V_{\mathrm{TH}}$ & $-36.4( \pm 3.8) \mathrm{V}$ & $-65.1( \pm 5.9) \mathrm{V}$ & $-52.9( \pm 4.4) \mathrm{V}$ \\
\hline & $I_{\mathrm{ON}} / I_{\mathrm{OFF}}$ & $564.3( \pm 69.6)$ & $3679.3( \pm 388.3)$ & $9443.9( \pm 883.8)$ \\
\hline & $\mu_{\mathrm{h}}$ & $2.4( \pm 0.5) \times 10^{-5}$ & $2.7( \pm 0.6) \times 10^{-4}$ & $9.8( \pm 2.1) \times 10^{-4}$ \\
\hline \multirow{3}{*}{ Р2ТВT } & $V_{\mathrm{TH}}$ & $-67.8( \pm 6.9) \mathrm{V}$ & $-37.8( \pm 4.9) \mathrm{V}$ & $-35.3( \pm 3.8) \mathrm{V}$ \\
\hline & $I_{\mathrm{ON}} / I_{\mathrm{OFF}}$ & $1321.9( \pm 128.3)$ & $5249.4( \pm 487.2)$ & $12574.5( \pm 895.3)$ \\
\hline & $\mu_{\mathrm{h}}$ & $2.5( \pm 0.5) \times 10^{-4}$ & $3.90( \pm 0.8) \times 10^{-4}$ & $1.5( \pm 0.3) \times 10^{-3}$ \\
\hline \multirow{3}{*}{ P2F2TBT } & $V_{\mathrm{TH}}$ & $-52.9( \pm 4.8) \mathrm{V}$ & $-48.6( \pm 3.7) \mathrm{V}$ & $-47.1( \pm 6.9) \mathrm{V}$ \\
\hline & $I_{\mathrm{ON}} / I_{\mathrm{OFF}}$ & $92.9( \pm 10.7)$ & $289.8( \pm 18.3)$ & $1710.8( \pm 138.2)$ \\
\hline & $\mu_{\mathrm{h}}$ & $8.1( \pm 1.5) \times 10^{-6}$ & $2.7( \pm 0.5) \times 10^{-4}$ & $1.49( \pm 0.3) \times 10^{-4}$ \\
\hline \multirow{3}{*}{ РBT2T2FB } & $V_{\mathrm{TH}}$ & $-59.4( \pm 5.4) \mathrm{V}$ & $-60.5( \pm 6.9) \mathrm{V}$ & $-65.5( \pm 7.1) \mathrm{V}$ \\
\hline & $I_{\mathrm{ON}} / I_{\mathrm{OFF}}$ & $45.0( \pm 3.9)$ & $95.1( \pm 10.7)$ & $237.5( \pm 18.3)$ \\
\hline & $\mu_{\mathrm{h}}$ & $2.2( \pm 0.5) \times 10^{-5}$ & $4.2( \pm 0.9) \times 10^{-5}$ & $1.7( \pm 0.4) \times 10^{-4}$ \\
\hline
\end{tabular}




\section{References}

R1. J. Choi, K.-H. Kim, H. Yu, C. Lee, H. Kang, I. Song, Y. Kim, J. H. Oh, B. J. Kim, Chem. Mater. 2015, 27,5230 .

R2. S. Subramaniyan, H. Xin, FS. Kim, SA. Jenekhe, Macromolecules, 2011, 44, 6245.

R3. C. J. Mueller, E. Gann, C. R. McNeill, M. Thelakkat, J. Mater. Chem. C. 2015, 3, 8916.

R4. J. Jo, J. Jung, H, Wang, P. Kim,T, P. Russell, W, Jo, Chem. Mater. 2014, 26, 4214.

R5. X. Chen, Z. Zhang, Z. Ding, J. Liu, L. Wang, Angew. Chem. Int. Ed .2016. 55, 10376. 